\title{
Case Study on Enterprise Behavior Considering Cluster Brand
}

\author{
Binbin He \\ Yancheng Party Institute of CCP, Yancheng, China \\ Email: hbbokok@163.com
}

How to cite this paper: He, B.B. (2019) Case Study on Enterprise Behavior Considering Cluster Brand. American Journal of Industrial and Business Management, 9, 1908-1914.

https://doi.org/10.4236/ajibm.2019.910124

Received: September 27, 2019

Accepted: October 21, 2019

Published: October 24, 2019

Copyright (c) 2019 by author(s) and Scientific Research Publishing Inc. This work is licensed under the Creative Commons Attribution International License (CC BY 4.0).

http://creativecommons.org/licenses/by/4.0/

\begin{abstract}
The industrial cluster brand is the carrier of the regional economic development. The Zhejiang economy is characterized by industrial clusters. At the same time of rapid economic development, some cluster brands featuring local industries have emerged. This paper analyzes the brand building of mature traditional industry clusters through the case of "Jinhua Ham" industrial cluster. Finally, it puts forward the policy construction of brand building of industrial clusters.
\end{abstract}

\section{Keywords}

Industry Cluster, Cluster Brand, Case Study

\section{Introduction}

Clusters brand is piled up by good reputation, because of the long-term good quality, comprehensive service, trustworthy behavior and criterion operation of the company within cluster. The formation of cluster brand has a positive effect on the enterprise within the industrial cluster; the consumers show their trust and loyalty to manufacturers of whom produce similar products in cluster with good brand. For the consumer, it is a guarantee of quality and reputation, and can reduce the costs and risks of consumer purchases; for enterprises in the cluster, the cluster brand benefits are generated by enjoying the cluster brand's intangible assets, which bring the brand effect to enterprises in the cluster. Cluster brand is the unity of consumer psychology and the value orientation of the spiritual values.

Domestic and foreign scholars did some research on the cluster brand and regional brand, found that its essence was the "cluster brand" [1]. It mainly concentrated in five aspects: 1) The definition and connotation of the cluster brand: 
the cluster band was regarded as the shared resource's affection to cluster competitiveness, while the heterogeneous resource is the basis of competitive advantage [2]. Xia Zengyu (2003) defined regional brand as a comprehensive reflection of the collective behavior of enterprise brand in a certain area, which formed a good reputation of an industry or a product in the regional [3]. Shao Jianping (2008) considered that the regional brand is the development product of the regional economy, culture, geography and natural resources which act on one another [4]. Thus industrial cluster brand can be defined as: the sign of a cluster differed from other clusters is the enterprises' long-term standardized management with good quality, comprehensive service, accumulated good reputation. So the industrial cluster brand represents the potential competitiveness and profitability of enterprises in cluster, and is known for their consumers, which led to the trust and loyalty of consumers to all manufacturers produce similar products of the enterprises in cluster. 2) Cluster brand is beneficial to improve the competitiveness of the cluster and its enterprises. Stuart (2002) thought that the implementation of the regional brand strategy in industrial cluster is an important way to enhance the competitiveness of developing countries [5]. Yang Jianmei (2006) described the brand structure of the industry cluster using the weighted average method, and analyzed the cluster brand structure's effect on the competitiveness of the cluster [6]. 3) The development and implementation methods of the cluster brand. E. Richard (2006) pointed out that setting up a system of knowledge capital is an important factor in the construction process of cluster brand [7]. Wu Chuanqing (2009) built the statistical and monitoring index system of industrial cluster brand from two levels: the enterprise brand evaluation and overall brand evaluation of industrial cluster [8]. Li Dalei (2008) built the development pattern model of cluster brand, using the empirical analysis method to test the model hypothesis [9], and he also made quantitative analysis and scientific verification on the components of cluster brand construction. 4) To the enterprises in cluster, cluster brand plays an important role and has influence on their behavior. Zhao Jing (2012) considered the enterprise brand in the study of the cluster brand, studied how the cluster brand affects the enterprise's brand value, by using the signalling theory [10]. Liu Qin (2004) adopted the game theory to study how the cluster brand affects the enterprises' decision-making in cluster [11]. Zhao Guanghua (2009) analyzed the evolution mechanism and improving path of industrial cluster brand, and built a rapid development diamond model of the clustered enterprises [12]. 5) From the viewpoint of the consumers, cluster brand has a positive or negative impact on consumers for the different types of cluster. Bi Nan (2009) used empirical analysis to indicate that the cluster brand has a significant impact on the product perceived quality of consumers under certain conditions. At the same time, the formation of the cluster brand affected by the enterprises brand within the cluster, the economic development of the cluster area [13]. Niu Yongge (2011) used data analysis to find that as for some industrial clusters, cluster brand has a negative effect on consumers, which is not 
suitable to develop cluster brand; while some industrial cluster is appropriate for developing cluster brand [14].

Therefore, scholars believed that the cluster brand played an important role to the enterprises in cluster and regional economic development, and the shaping and cultivating of cluster brand would be affected by the behavior of consumers and enterprises. However, cluster brand is public goods for the enterprises in cluster, any enterprises in cluster can share the benefits which cluster brand bring. It also has the characteristic of non-exclusive and non-competitive. At the same time, for the new enterprises, the marginal social cost of the cluster brand in cluster is zero. Whether the cluster enterprises make contribution to the maintenance of the cluster brand or not, they can benefit from the public goods. So cluster brand need to be maintained by all the members in cluster, while maintaining the cluster brand has some cost, such as honest and trustworthy behavior leads to the reduction of opportunism behavior income, and improving quality makes cost increased and so on. However, enterprises in cluster can also choose to hitchhike instead of maintaining cluster brand. Which factors related to the decision-making of whether the enterprises in cluster maintain the cluster brand or not? How to promote enterprises in cluster to maintain the cluster brand consciously, which plays an important role in enhancing the competitiveness of enterprises and clusters and even promoting regional economic development. The shaping and cultivating of cluster brand need a long-term. Because of the enterprises' bounded rationality, under the circumstance of long-term game among enterprises in cluster, how does the behavior of maintaining cluster brand evolved? The brand building of industrial clusters not only requires the conscious maintenance of enterprises, but also the support of the government and industry associations. The next article will take Jinhua Ham Industrial Cluster as an example to analyze the specific work of industrial cluster brand construction.

\section{Case Study-Analysis of the Industrial Cluster of Jinhua Ham}

\subsection{Overview of the Development of "Jinhua Ham" Industrial Cluster}

China's cultural history is profound, and under the continuous culture of a long time, many brands named "Place of Origin + Product" have been formed, such as Jinhua Ham, Jingdezhen Porcelain, Yangcheng Lake Hairy Crab, Yunnan Pu'er and Wuyou Drunk Snail. Most of the above brands rely on the resources and cultural advantages of the brand location. The quantity and quality of the products are superior to those of other regions. In addition, in the long history and cultural heritage, they have also deeply imprinted the hearts of consumers. This brand has a relatively long-lasting commercial value that other brands do not have and has a high reputation in the market.

Jinhua Ham is a typical industrial cluster brand named after the common name 
of the place of origin goods, and has enjoyed a very high reputation for a long time. Jinhua Ham has a very obvious agglomeration phenomenon. By the end of 2018, there were 132 ham production enterprises in Jinhua City. In terms of market competitiveness, Jinhua Ham is one of the traditional Chinese specialty food brands, which is well-known in the national ham market $75 \%$ share. Figure 1 shows the source of competitive advantage of Jinhua Ham Industrial Cluster.

\subsubsection{Supporting Industry Scale Farms}

As environmental protection, capital, land and other industries the threshold significantly increased, the advantages of large-scale fields compared to other farming entities continue to expand. Strengthening large-scale farming is the future development of the ham industry cluster.

\subsubsection{Cluster Competitive Advantage}

On the one hand, the excellent brand effect generated by the cluster brand can enhance the competitiveness of the cluster and promote the long-term development of the cluster; on the other hand, the cluster brand can counter the construction of product brands and corporate brands in the cluster and promote the development of industrial clusters.

\subsubsection{Leading Companies and Competition}

Leading the development of the cluster industry with the leader will help improve the overall innovation level of the cluster. Make full use of the agglomeration effect of leading enterprises on talents and resources to help other enterprises in the cluster to improve their independent innovation capabilities.

\subsubsection{Market Demand Great Potential}

The fermented meat products produced by large-scale production of fermenting agents in Europe and the United States are widely accepted by consumers and have a huge market capacity. In China, the systematic research and industrialized production of fermented meat products started late, but it has broad market development prospects.

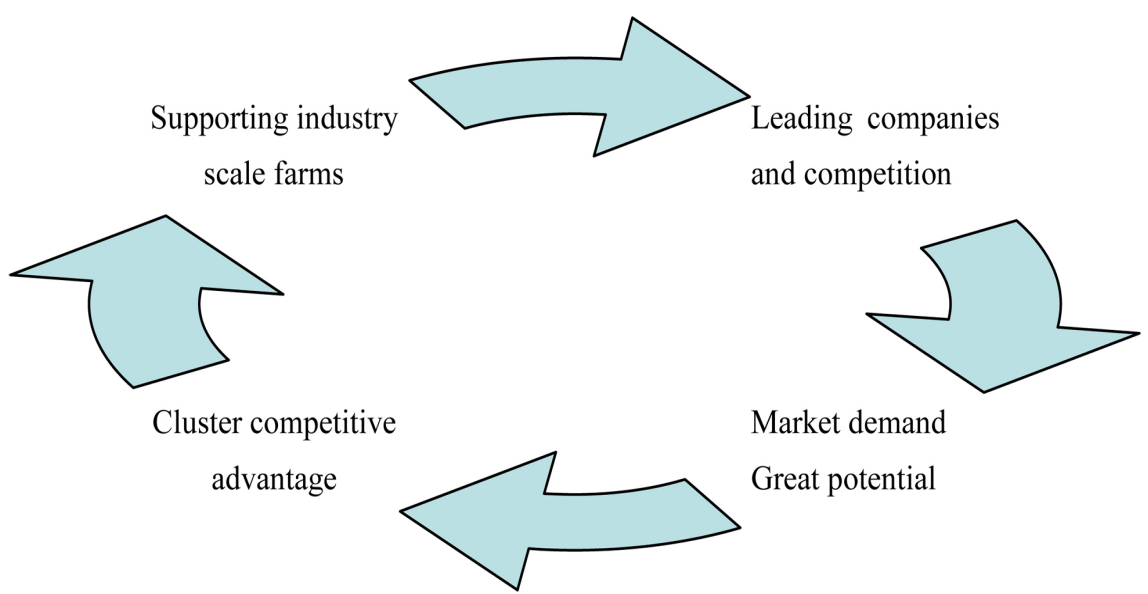

Figure 1. Sources of competitive advantage of the "Jinhua Ham" industrial cluster. 
According to the above introduction, it can be found that the "Jinhua Ham" cluster plays a very important role in the local economic development. The Jinhua Ham Cluster has a good corporate competition mechanism and a perfect supply model. Many homogeneous enterprises are clustered in the cluster. There are sufficient breeding industries to provide raw materials for production. The Jinhua area already has a relatively complete industrial chain. The clusters formed by related industries such as ham industry constitute an important factor driving the economic development of the region.

\section{2. "Jinhua Ham" Brand Crisis}

In 2003, two ham companies in the Jinhua area soaked them with dichlorvos when they produced ham in the off-season. When the incident was exposed, the social response was strong. Consumers adopted a distrustful attitude towards all Jinhua ham producers. The ham brand that has been in existence for more than a thousand years has been devastated. Due to the influence of public opinion and the general popularity of Jinhua Ham, this incident has left a huge impact on consumers. In a short period of time, the sales of ham began to drop sharply, even from high-quality ham from formal channels. It has also been affected. According to relevant survey statistics, the decline in ham sales has reached about $70 \%$ for a period of time [15] (analyzes on regional brand crisis of collective punishment by Journal of Jinhua College of Profession and Technology). Many supermarkets in Zhejiang have squeezed a large number of Jinhua hams. In the same year, enterprises producing ham in Jinhua area suffered heavy losses, continuing for thousands. The brand of the rest of the year has also been cast a shadow.

Due to the huge impact of the "Jinhua Ham" product quality incident, individual enterprises cannot respond effectively. This requires the government to take effective measures. The Jinhua Municipal Government has adopted a number of policies to deal with the brand crisis, including crisis public relations activities and development, industry special rectification activities. First of all, vigorously promote the ham products in the formal channels, prompt consumers to understand the brand of Jinhua Ham, severely crack down on the two companies involved in the case, Yongtai and Xuchun, destroy the unqualified ham, and carry out special rectification of the whole industry to ensure the time. The event no longer occurs.

With the strong support of the government, in 2004, "Jinhua Ham" returned to the supermarkets and other markets and regained its reputation among the consumer groups. Obviously, the government has played an important role in protecting the "Jinhua Ham" brand. At the same time, the industry association has also played an important role in assisting the Jinhua Municipal Government in the special rectification of the whole industry.

From this case, the enterprises in the industrial clusters will have a huge negative impact on the industry in the cluster by external diseconomy of the cluster brand. The credibility crisis that occurs within the industrial cluster is likely to 
escalate into a regional credibility crisis. Individual free-riding companies generally have the ability to solve problems. This requires the government to proceed from the overall situation and timely regulate the trading behavior within the cluster. Facts have proved that local government supervision and other activities have a great effect on regulating corporate transactions and enhancing the reputation of cluster brands.

\section{The Enlightenment Brought by the Case of "Jinhua Ham"}

According to the Jinhua Ham industry cluster case, it can be concluded that the government is improving the brand performance of local industrial clusters.

1) Cluster brand is the focus of local government regulation. The industrial cluster brand is different from the private brand of the general enterprise. It can be regarded as a collective brand with the characteristics of public products. The ownership of the property rights has diversified characteristics. The main body of the property rights supervision should not only include the government and industry of the industrial cluster. Associations or chambers of commerce should also improve the self-discipline of cluster enterprises. The quality supervision of cluster enterprises is weak or lack of supervision. This is because profit-seeking enterprises lack motivation in supervision and need external factors to guide them. Therefore, the government and industry associations should guide enterprises in different categories and in a unified manner.

2) Local governments should play a leading role in the process of cluster brand supervision. Obviously, local governments must be the brand supervision of industrial clusters because of the advantages of local governments in the supervision of the use of industrial cluster brands and the supervision of cluster product quality, such as organizational resources, policy resources and technical resources, as well as the authority and impartiality of public power. The leader, the government should carry out special product management around the actual problems faced by local clusters in the handling of product quality crisis, not only to ensure the government's main position in the maintenance of industrial cluster brands, but also to strengthen the coordination mechanism between enterprises and enterprises in the cluster.

3) Enterprises should pay attention to cultivating their own corporate culture, cultivate employees' sense of identity through the training and education of employees, and deal with food safety issues as a matter of life and death of the company, on the basis of ensuring the quality of products. Increase production scale, strengthen the company's own management level and ability to cope with risks, regard the company's reputation as an integral part of the company's core values, cultivate consumer loyalty to products, and strive to improve the competitiveness of its products.

\section{Conclusion}

This paper analyzes the cluster brand through the case of Jinhua Ham industry cluster. We found the government can increase the cluster brand publicity by 
regulating the behavior of enterprises and increasing brand awareness of the public, which can effectively promote the healthy and steady development of industrial cluster.

\section{Conflicts of Interest}

The author declares no conflicts of interest regarding the publication of this paper.

\section{References}

[1] Fu, Z.-P. (2004) Research on Small and Medium-Sized Enterprises Cluster Formation Mechanism. Zhongshan University Press, Guangzhou.

[2] Barney, J.B. (1991) Firm Resources and Sustained Competitive Advantage. Journal of Management, 17, 99-120.

[3] Xia, Z.-Y. and Xie, J. (2003) Regional Brand Developments Study-Case Example Research in the Wenzhou. China Industrial Economy, No. 10, 43-48.

[4] Shao, J.-P. and Ren, H.-L. (2008) Forming Mechanism and Utility Transmission of Regional Brand for the Northwest Regional Brand Cultivating Enlightenment. Science and Technology Management Research, No. 3, 133-134.

[5] Rosenfeld, S.A. (2002) A Guide to Cluster Strategies in Less Favored Regions. Conference of Regional Technology Strategies, Carrboro, 28-30.

[6] Yang, J.-M., Huang, X.-Z. and Zhang, S.-T. (2006) Brand Structure of Cluster and Its Effects on Competitiveness of Industrial Clusters. Science and Technology Management Research, No. 11, 37-39.

[7] Gold, E.R. (2006) Intellectual Architecture as Place Brand. Place Branding, 2, 220228.

[8] Wu, C.-Q. (2009) Research on Industrial Cluster Brand Monitoring Statistics Index. Management World, No. 11, 174-175.

[9] Li, D.-L. and Zhong, W.-Z. (2008) A Positive Study on the Transformation of the Mode of Industrial Cluster Brand. Journal of Business Economics, No. 8, 53-60, 67.

[10] Zhao, J., Guo, B. and Guo, H. (2012) Interaction between Cluster Brand and Enterprise Brand Based on Signaling Theory. China Soft Science, No. 3, 149-161.

[11] Liu, Q. and Chen, J.-X. (2004) Game Analysis on Risk of Enterprise Cluster Brand. Contemporary Finance \& Economics, No. 9, 69-72.

[12] Zhao, G.-H. and Ren, D.-K. (2009) The Mechanism and Path of Ascension for Industrial Cluster Brand. Science Press, Beijing.

[13] Bi, N. and Sun, L.H. (2009) An Experimental Research on the Impact of Cluster Branding Based on Product Perceived Quality. Management Review, 21, 52-60.

[14] Niu, Y.-G. and Zhao, P. (2011) Effects of Industrial Cluster Brand from Consumers' Perspective. Journal of Management Science, 24, 42-54.

[15] Chen, Y.Q., Ni, Z.J. and Chen, Z.X. (2005) Analysis of the Causes of the Regional Brand Bian Crisis-Taking the Jinhua Ham Brand Cluster Incident as an Example. Journal of Jinhua College of Profession and Technology, 5, 17-21. 